\title{
Inter-site and interpersonal diversity of salivary and tongue microbiomes, and the effect of oral care tablets [version 1;
}

\section{peer review: 3 approved]}

\author{
Hugo Maruyama (iD1, Ayako Masago², Takayuki Nambu1, Chiho Mashimo1, \\ Kazuya Takahashi², Toshinori Okinaga ${ }^{1}$ \\ 1Department of Bacteriology, Osaka Dental University, Hirakata, Osaka, 573-1121, Japan \\ 2Department of Geriatric Dentistry, Osaka Dental University, Hirakata, Osaka, 573-1121, Japan
}

\begin{tabular}{l}
\hline V1 First published: 17 Dec 2020, 9:1477 \\
https://doi.org/10.12688/f1000research.27502.1 \\
Latest published: 09 Apr 2021, 9:1477 \\
https://doi.org/10.12688/f1000research.27502.2 \\
\hline
\end{tabular}

\section{Abstract}

Background: Oral microbiota has been linked to both health and disease. Specifically, tongue-coating microbiota has been implicated in aspiration pneumonia and halitosis. Approaches altering one's oral microbiota have the potential to improve oral health and prevent diseases.

Methods: Here, we designed a study that allows simultaneous monitoring of the salivary and tongue microbiomes during an intervention on the oral microbiota. We applied this study design to evaluate the effect of single-day use of oral care tablets on the oral microbiome of 10 healthy individuals. Tablets with or without actinidin, a protease that reduces biofilm formation in vitro, were tested.

Results: Alpha diversity in the saliva was higher than that on the tongue without the intervention. The core operational taxonomic units (OTUs) common to both sites were identified. The salivary and tongue microbiomes of one individual tended to be more similar to one another than to those of other individuals. The tablets did not affect the alpha or beta diversity of the oral microbiome, nor the abundance of specific bacterial species.

Conclusions: While the salivary and tongue microbiomes differ significantly in terms of bacterial composition, they show inter-rather than intra-individual diversity. A one-day usage of oral care tablets did not alter the salivary or tongue microbiomes of healthy adults.

Whether the use of oral tablets for a longer period on healthy people or people with greater tongue coating accumulation shifts their oral microbiome needs to be investigated.

Keywords

oral care tablet, oral microbiome, actinidin, QIIME 2

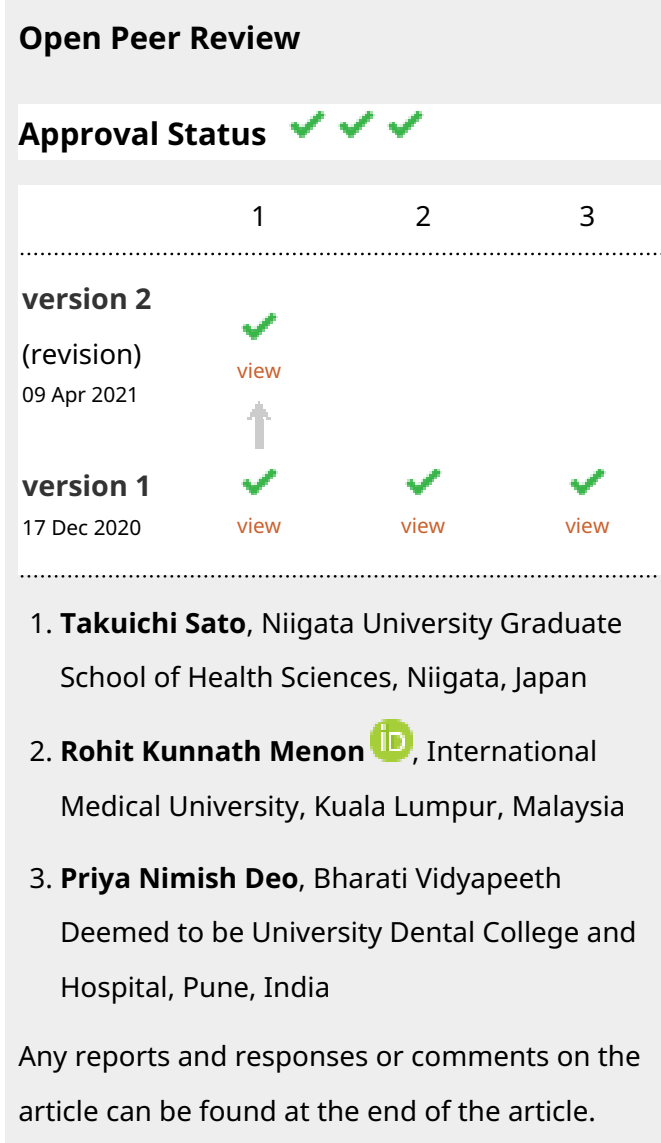


Corresponding author: Hugo Maruyama (maruyama@cc.osaka-dent.ac.jp)

Author roles: Maruyama H: Formal Analysis, Funding Acquisition, Investigation, Visualization, Writing - Original Draft Preparation, Writing - Review \& Editing; Masago A: Data Curation, Investigation, Project Administration; Nambu T: Conceptualization, Formal Analysis, Funding Acquisition, Methodology, Project Administration, Writing - Review \& Editing; Mashimo C: Conceptualization, Funding Acquisition, Writing - Review \& Editing; Takahashi K: Conceptualization, Funding Acquisition, Resources; Okinaga T: Resources, Supervision, Writing - Review \& Editing

Competing interests: No competing interests were disclosed.

Grant information: This work was supported by Japan Society for the Promotion of Science (JSPS) KAKENHI [17K15254 to HM, 20 K10285 to TN, $16 \mathrm{~K} 11876$ to CM, and 19K10473 to KT].

The funders had no role in study design, data collection and analysis, decision to publish, or preparation of the manuscript.

Copyright: @ 2020 Maruyama $\mathrm{H}$ et al. This is an open access article distributed under the terms of the Creative Commons Attribution License, which permits unrestricted use, distribution, and reproduction in any medium, provided the original work is properly cited.

How to cite this article: Maruyama $\mathrm{H}$, Masago A, Nambu T et al. Inter-site and interpersonal diversity of salivary and tongue microbiomes, and the effect of oral care tablets [version 1; peer review: 3 approved] F1000Research 2020, 9:1477

https://doi.org/10.12688/f1000research.27502.1

First published: 17 Dec 2020, 9:1477 https://doi.org/10.12688/f1000research.27502.1 


\section{Introduction}

Oral microbiota is a collection of microorganisms that reside in the oral cavity. It has been linked to the promotion of both health and disease ${ }^{1,2}$. Among the different tissues in the oral cavity, the tongue is considered a dominant source of oral microbial populations ${ }^{3,4}$. Further, tongue coating is proposed to cause oral malodor ${ }^{5}$ or, upon sudden dissociation, aspiration pneumonia in elderly people with impaired defense mechanisms ${ }^{6,7}$. In addition, the tongue coating is a risk indicator of aspiration pneumonia in edentate individuals ${ }^{8}$.

A variety of methods to reduce tongue coating have been developed and tested to reduce oral malodor ${ }^{9,10}$. Mechanical removal of the tongue coating using tongue brushes or tongue cleaners is one such popular method ${ }^{9,11}$. Other methods include using antimicrobials, e.g., in gels or mouthwashes, or using oral tablets $^{10,12}$.

The tongue microbiota in elderly individuals has been classified into several types with characteristic bacterial composition. These types correlate with the risk to aspiration pneumonia ${ }^{4,13}$. Therefore, methods that could alter the tongue microbiota to a healthy microbiota type could contribute to oral health. We have previously reported that tongue brushing does not alter the alpha or beta diversity of oral microbiota in healthy adults ${ }^{14,15}$. By contrast, according to a recent study, the use of oral care tablets decreases the amount of volatile sulfur compounds (VSCs) produced by bacteria $^{16}$. Further, oral care tablets that contain actinidin, a cysteine protease found in kiwifruit, reduce oral biofilm formation in vitro ${ }^{12}$. However, it is not clear whether these interventions affect the oral microbiota as a whole or the abundance of specific bacteria.

In the current study, we examined the effect of oral care tablets with and without actinidin on the salivary and tongue microbiomes of healthy individuals. We also investigated the diversity of the salivary and tongue microbiomes, and interpersonal microbiome diversity. We show that alpha diversity of the salivary microbiome was greater than that of the tongue microbiome; that an individual's salivary and tongue microbiomes were more similar to one another than to those of another individual; and that the oral care tablets did not affect the oral microbiomes in the population tested. These findings add to the knowledge of the interpersonal diversity and dynamics of the oral microbiota in humans.

\section{Methods}

Ten healthy adults participated in the study, with three different treatments tested: two different types of oral tablets (with or without protease), and a negative control (no tablet). For the tablet treatments, the saliva and tongue coating were collected between October 2016 and November 2017 at participants' home (mainly in Osaka, Japan, and in some cases nearby prefectures). DNA extraction and data analysis were conducted at Department of Bacteriology, Osaka Dental University (Hirakata, Japan).

\section{Participants}

Participants were recruited from faculty members and graduate students working at the Osaka Dental University hospital, as well as from dentists who were acquainted with an author of this study. Ten healthy volunteers (6 males and 4 females; age: $27-60$ years $[39.8 \pm 3.1$ (mean \pm SD) $]$ ) were enrolled in the study and were anonymized randomly as A-G, O, Q, and R (Table 1). The inclusion criteria were as follows: healthy men and women over 20 years of age. The exclusion criteria were as follows: daily smoking; treatment with local or systemic antibiotics within 1 month prior to the study; and allergy to kiwifruit. The method and objective of this study were explained to the participants, who provided written informed consent before participating. The Osaka Dental University Medical Ethics Committee approved this study (approved on 3/31/2015; approval number 110864) and the investigations were conducted following the rules of the Declaration of Helsinki. The committee did not consider the study to be interventional in nature and therefore is not a clinical trial.

\section{Oral tablets}

Two types of oral care tablets for tongue cleaning were tested in the current study. One type contained actinidin, a cysteine protease extracted from kiwifruit ("protease tablet") and the other did not ("plain (placebo) tablet"). Both tablets were provided by Ezaki Glico Co., Ltd (Osaka, Japan). The protease tablets are identical to those marketed as BREO EX (Ezaki Glico Co.). Tablet composition was described previously ${ }^{12}$. To use the tablets, the participants placed one tablet on the dorsum of the tongue and waited until it dissolved naturally. One tablet takes approximately 5-7 min to completely dissolve.

Study design

The study design is illustrated in Figure 1. The tongue tablet experiment was a placebo-controlled double-blind crossover

\section{Table 1. Demographic data of the participants.}

\begin{tabular}{|l|l|l|l|}
\hline Participant & Age & Gender & Ethnicity \\
\hline A & 50 & Female & Asian \\
\hline B & 45 & Male & Asian \\
\hline C & 42 & Male & Asian \\
\hline D & 35 & Female & Asian \\
\hline E & 60 & Female & Asian \\
\hline F & 28 & Male & Asian \\
\hline G & 27 & Male & Asian \\
\hline O & 27 & Female & Asian \\
\hline Q & 39 & Male & Asian \\
\hline R & 45 & Male & Asian \\
\hline
\end{tabular}


Tablet treatments

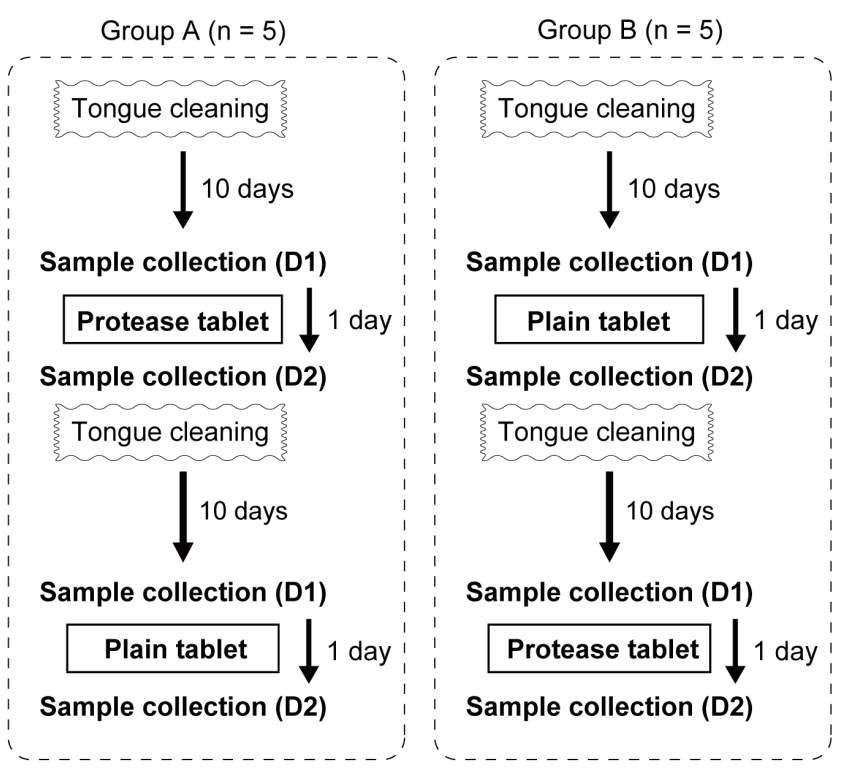

(a)
No tablet treatment (control)

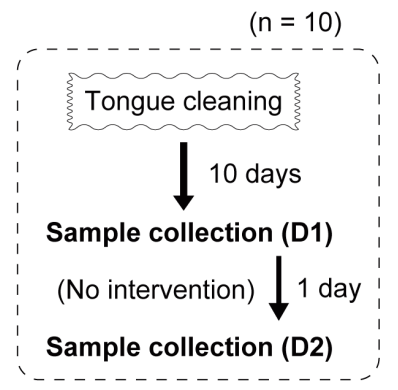

(b)

Figure 1. Schematic representation of the study design and trial schedule. (a) Ten participants were randomly divided into two groups for the tongue tablet trials. (b) Control (no tablet usage) treatment. The duration between the tablet treatments and the control experiment ranged from 10-60 days.

study. The 10 participants were randomly divided into 2 groups of 5 participants each, by using computer-generated random numbers. All participants performed an initial tongue cleaning (by brushing) at the beginning of the study. The participants were asked not to eat, drink, or perform oral cleaning before each sampling. After a washout period of 10 days during which the participants did not perform any tongue cleaning, they collected their saliva and tongue coating into separate containers in the morning immediately after waking up (sample D1). Then, the participants in each group took tablets, with or without the protease. The participants and the researchers who analyzed the data were not informed about the tablet types given to the participants. The participants were asked to use the tablet three times on the day of the experiment-in the morning (between 9-12 am), in the afternoon (1-4 pm), and in the evening (7-10 pm) - taking one tablet each time. The following morning, the participants collected their saliva and tongue coating separately immediately after waking up (sample D2). After a washout period of 10 days, the participants took the other type of tablet that they had not previously received, and collected the saliva and tongue samples as before. Control experiments (no tablet usage) were conducted with the same participants, after they conducted treatment using the tablets. The duration between the tablet treatments and the control experiment ranged from 10-60 days, depending on the participant. In these experiments, after an initial tongue cleaning and a 10-day washout period, all participants collected samples on two consecutive days (D1 and D2) without taking any tablet in between.

\section{Sample collection}

The saliva and tongue-coating samples were collected immediately after the participants woke up, in the morning of the day of tongue cleaning by tablet (D1) and the next morning (D2). The participants first collected $3 \mathrm{~mL}$ of saliva in a $25-\mathrm{mL}$ sterile plastic tube. The tongue coating was collected by scrubbing the tongue with a swab and then soaking the tip of the swab in $0.6 \mathrm{~mL}$ of phosphate-buffered saline (PBS(-); Wako Pure Chemical Industries, Ltd., catalogue number 166-23555) to suspend the coating. Because this collection method involves scrubbing the tongue with a swab, the tongue coating was collected from the left half of the tongue for D1 and from the right half of the tongue for D2, to minimize the carryover effects of scrubbing. The collected samples were maintained at $4^{\circ} \mathrm{C}$ for up to 1 day and transported to the laboratory. The saliva samples $(3 \mathrm{~mL})$ were homogenized by repetitive pipetting. Then, $0.5-\mathrm{mL}$ aliquots were transferred to sterile tubes. The saliva $(0.5 \mathrm{~mL})$ and tongue-coating $(0.6 \mathrm{~mL})$ samples were then centrifuged at $10,000 \times g$ for $4 \mathrm{~min}$. The supernatant was discarded and the pellet was stored at $-20{ }^{\circ} \mathrm{C}$ until DNA extraction. All samples were frozen no later than on the day of D2 sampling.

\section{DNA extraction and library construction}

Bacterial DNA was extracted from the pellets via chemical and mechanical lysis using a QIAamp UCP Pathogen Mini kit (QIAGEN, catalogue number 50214), as previously described ${ }^{17,18}$. Briefly, thawed pellets were immediately suspended in 
$0.5 \mathrm{~mL}$ of ATL buffer containing the optional DX reagent, transferred to a Pathogen Lysis Tube S, and then homogenized using a Mixer Mill MM 301 (Retsch) for $3 \mathrm{~min}$ at a vibrational frequency of $30 \mathrm{~Hz}$. The manufacturer's protocol was followed thereafter to complete the DNA purification. DNA was eluted in $50 \mu \mathrm{L}$ of the AVE buffer (QIAGEN). DNA concentration was determined using a Quantus fluorometer (Promega) and a Qubit dsDNA BR Assay kit (Thermo Fisher Scientific, catalogue number Q32850). DNA was stored at $-80{ }^{\circ} \mathrm{C}$ until use.

Bacterial 16S ribosomal DNA amplification and library construction were performed according to the 16S Metagenomic Sequencing Library Preparation guide supplied by Illumina (part No. 15044223_B), as previously described ${ }^{17}$. The V3-V4 region of the 16S ribosomal RNA gene was amplified by polymerase chain reaction (PCR) with a thermal cycler MJ-Mini (Bio-Rad Laboratories), using primers 341F (5'-TCGTCGGCAGCGTCAGATGTGTATAAGAGACAGCCTACGGGNGGCWGCAG-3') and 806R (5'-GTCTCGTGGGCTCGGAGATGTGTATAAGAGACAGGGACTACHVGGGTWTCTAAT-3') (custom-synthesized by Invitrogen), and Premix Ex Taq Hot Start Version (Takara Bio, catalogue number RR030A). The thermal cycling conditions were initial denaturation at $98{ }^{\circ} \mathrm{C}$ for $10 \mathrm{~s}$, followed by 25 cycles at $98{ }^{\circ} \mathrm{C}$ for $10 \mathrm{~s}, 55^{\circ} \mathrm{C}$ for $30 \mathrm{~s}$, and $72{ }^{\circ} \mathrm{C}$ for $1 \mathrm{~min}$ (the first PCR step). The underlined nucleotides served as primer sequence parameters to extract the V3-V4 region for feature classifier training (see next section). The amplicons were purified using AMPure XP beads (Beckman Coulter, catalogue number A63880). Sequencing adapters containing 8-bp indices were incorporated at the 3'- and 5'-ends of the purified amplicons during a second PCR step. The amplicons were again purified using the AMPure XP beads, and then quantified using a Quantus fluorometer (Promega) and a Qubit dsDNA HS Assay kit (Life Technologies, catalogue number Q32851). After pooling equimolar amounts of the amplicons, $5 \%$ of an equimolar amount of PhiX DNA (PhiX Control v3, Illumina, catalogue number FC-110-3001), was added. The obtained library was pair-end sequenced at $2 \times 250$ bp using a MiSeq Reagent Kit v2 (Illumina, catalogue number MS-1022001) and the Illumina MiSeq platform. Sequencing was performed over seven independent runs at the Oral Microbiome Center (Takamatsu, Japan), followed by demultiplexing. Raw nucleotide sequences are available at DDBJ/EMBL-EBI/NCBI database under the accession number DRA010849.

\section{Sequence processing and data analysis}

Demultiplexed paired-end sequences were processed using QIIME 2 (v.2020.2) and its associated plugins ${ }^{19}$ in a Docker container. Sequences obtained from independent Miseq runs were denoised separately using DADA2 (via q2-dada2) ${ }^{20}$ applying previously-optimized parameters $^{14}$ (trim-left-f $=20$; trim-left-r $=20$; trunc-len-f and trunc-len- $r$ were set between 241 and 248 depending on the sequence quality; other parameters followed the default settings, including chimera-method $=$ "consensus"). The resulting exact amplicon sequence variants (ASVs) were merged (via q2-feature-table). For taxonomy assignment to each ASV, a naïve Bayes taxonomy classifier trained (via q2-feature-classifier) ${ }^{21}$ on the V3-V4 region of the 16S rRNA sequences in the expanded human oral microbiome database (eHOMD; v.15.2) 22 $^{2}$ was used. All ASVs were aligned using MAFFT $^{23}$ and used to construct a phylogeny with FastTree 2 (via q2-phylogeny) ${ }^{24}$. Sample metadata format was validated using the cloud-based tool Keemei ${ }^{25}$.

Alpha diversity was assessed by calculating the number of observed features (ASVs) and the Shannon index (via q2-diversity), after samples were subsampled without replacement (rarefied) to 40,000 sequences per sample. The non-parametric Kruskal-Wallis test was used to test for significant differences in alpha diversity between sample groups $(P<0.05)$.

Beta diversity was computed based on the unweighted UniFrac distance ${ }^{26}$ (via q2-diversity) and visualized as threedimensional principal coordinate analysis ( $\mathrm{PCoA}$ ) plots using EMPeror (via q2-emperor) ${ }^{27}$. Permutational analysis of variance (PERMANOVA) $^{28}$ was used to test the significant difference in bacterial composition among samples $(P<0.05)$. Differential abundance of bacterial taxonomic groups was tested using the analysis of composition of microbiomes (ANCOM) (via q2-composition) $)^{29}$.

Clustered operational taxonomic unit (OTU) table was also used to calculate the beta diversity and differential abundance of specific taxonomic groups. Open-reference clustering was chosen for a high-quality taxonomic assignment to a curated database $^{30}$. Here, the entire ASVs were clustered into OTUs by open-reference picking (via q2-vsearch) ${ }^{30}$ using the V3-V4 region of $16 \mathrm{~S}$ rRNA sequences in the eHOMD v.15.2 as a reference, with $99 \%$ identity threshold. A phylogenetic tree was constructed as described above. The R package treeio (v.1.12.0) ) $^{31}$ was used to change the OTU names within the phylogenetic trees, as per the requirement for the Rhea package (the software disallows OTU names starting with a number). Multidimensional scaling (MDS) based on generalized UniFrac distance ${ }^{32}$ was performed to examine the difference in microbial composition among samples, using Rhea pipeline (v.1.1.3) (33 $^{33}$ For differential abundance analysis, "Serial group comparisons" in Rhea was performed (abundance_cutoff $=0.2$; prevalence cutoff $=0.3$; max_median_cutoff $=1$ ) with significance cutoff of $\mathrm{p}<0.05$ in Kruskal-Wallis test.

Box plots that show the alpha diversities were generated using $\mathrm{R}$ packages ggplot2 (v.3.3.2) and ggpubr (v.0.4.0), with data retrieved from QIIME 2 artifacts using qiime2R (v.0.99.31). Heatmaps and box plots that show the relative abundance of microbial taxonomies, and a Venn diagram that show core OTUs, were generated using ampvis2 (v.2.6.5) $)^{34}$ R (v.4.0.2) and RStudio (v.1.3.959) were used for all analyses. Software, plugins, and $R$ packages used in this study is listed in Table 2.

\section{Results}

\section{Study overview}

The study design is illustrated in Figure 1. Ten healthy adults participated in the study, with three different treatments tested: two different types of oral tablets (with or without protease), and a negative control (no tablet). For the tablet treatments, the saliva and tongue coating were collected before (D1) and after (D2) the intervention. Overall, 116 samples were collected [10 participants, three treatments, two sites (the saliva and tongue), and two sampling time points (D1 and D2), with four 
Table 2. List of software, plugin, $R$ packages used in the study.

\begin{tabular}{|l|l|l|}
\hline Name & Version & URL \\
\hline QIIME 2 & 2020.2 & https://qiime2.org/ \\
\hline Keemei & & https://keemei.qiime2.org/ \\
\hline R & 4.0 .2 & https://www.r-project.org/ \\
\hline Rstudio & 1.3 .959 & https://rstudio.com/ \\
\hline Rhea & 1.1 .3 & https://github.com/Lagkouvardos/Rhea \\
\hline ampvis2 & 2.6 .5 & https://madsalbertsen.github.io/ampvis2/index.html \\
\hline treeio & 1.12 .0 & http://bioconductor.org/packages/release/bioc/html/treeio.html \\
\hline qiime2R & 0.99 .31 & https://github.com/jbisanz/qiime2R \\
\hline ggplot2 & 3.3 .2 & https://ggplot2.tidyverse.org/ \\
\hline ggpubr & 0.4 .0 & https://github.com/kassambara/ggpubr \\
\hline
\end{tabular}

QIIME 2 plugins are not listed here because they are associated with specific version of QIIME 2.

samples excluded because of insufficient amount of extracted DNA]. The sample metadata are provided as underlying data (Table S1) $)^{35}$.

DNA was extracted from each sample and the V3-V4 region of the 16S rRNA gene was PCR-amplified. The amplicons were paired-end sequenced using the Miseq platform. After quality control and error correction using DADA2, 11,260,102 reads corresponding to 5342 ASVs were obtained. Per-sample median was 89,923 , with a maximum of 186,864 and a minimum of 46,422 . Open-reference clustering, using the curated 16S rRNA sequences in the eHOMD v.15.2 database as the reference (at 99\% identity threshold), grouped the sequences into 1210 OTUs. Either the full or clustered table was analyzed further, depending on the type of analysis performed, as described. The clustered OTU table is provided as underlying data $\left(\right.$ Table S2) ${ }^{36}$.

\section{Inter-individual diversity of the salivary and tongue} microbiomes

We first analyzed the microbiome of the saliva and tongue coating, to determine the baseline for the study. In total, 30 D1 samples (10 participants, three independent treatments) each of the saliva and tongue coating were analyzed. Alpha diversity of the tongue microbiome was significantly lower than that of the salivary microbiome, using both the number of observed ASVs $(P=8.9 \mathrm{e}-7$, Kruskal-Wallis test) (Figure 2a) and Shannon index $(P=2.0 \mathrm{e}-7$, Kruskal-Wallis test $)$ as measures (Figure 2b). This was consistent with a previous report ${ }^{3}$. The difference in diversity is probably associated with the tongue acting as a specialized niche for specific microorganisms, and the saliva containing a mixture of microbiota from different sites in the oral cavity. Interestingly, the number of observed ASVs varied among the individuals, ranging from approximately 170 to 325 in the saliva $(P=0.027$, Kruskal-Wallis test), and from approximately 125 to 225 in the tongue coating
$(P=0.022)$ (Figure 2c), suggesting a difference in oral microbiome among individual.

We next assessed the differences in bacterial composition among samples (beta diversity) (Figure 3). A significant difference between the salivary and tongue microbiomes was detected both in PCoA based on unweighted UniFrac distances using the full ASV table ( $P=0.001$, PERMANOVA) (Figure 3a) and MDS based on generalized UniFrac distances ${ }^{32}$ using the clustered OTU table ( $P=0.003$, PERMANOVA) (Figure 3c).

The data also revealed a significant difference between individual microbiomes (Figure $3 \mathrm{~b}$ and $3 \mathrm{~d} ; P=0.001$, PERMANOVA using the clustered OTU table). As indicated by the plots in Figure 3, the similarity of the salivary and tongue microbiomes within an individual was greater than the similarity of the salivary or tongue microbiomes between individuals. This suggests there is stability in an individual's oral microbiome, at least within the relatively short time period of the study (several weeks). This observation is consistent with earlier studies that highlight the stability of an individual's oral microbiome ${ }^{37}$.

\section{Differential abundance of bacterial taxonomic groups} in the salivary and tongue microbiomes

The abundances of bacterial taxonomic groups at the genus or species levels in the salivary and tongue microbiomes, determined by the analysis of D1 samples from the three treatments and based on the clustered OTU table are summarized in Figure $4 \mathrm{a}$ and $4 \mathrm{~b}$. The eight most abundant genera were common to the salivary and tongue microbiomes, accounting for nearly $80 \%$ of both microbiomes $(78.2 \%$ in the saliva and $80.9 \%$ in the tongue). These genera were Prevotella (18.4\% and $23.5 \%$, respectively), Veillonella ( $9.0 \%$ and $12.6 \%$, respectively), Neisseria $(11.6 \%$ and $9.9 \%$ respectively), Haemophilus $(10.9 \%$ and $8.6 \%$, respectively), Streptococcus $(9.4 \%$ and $6.1 \%$, respectively), Alloprevotella $(8.2 \%$ and $5.9 \%$, respectively), 
Participant $\bullet A \bullet C \bullet E \bullet G \bullet Q$

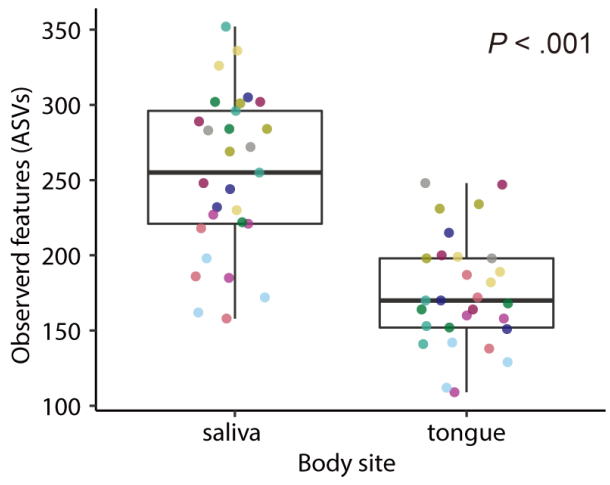

(a)

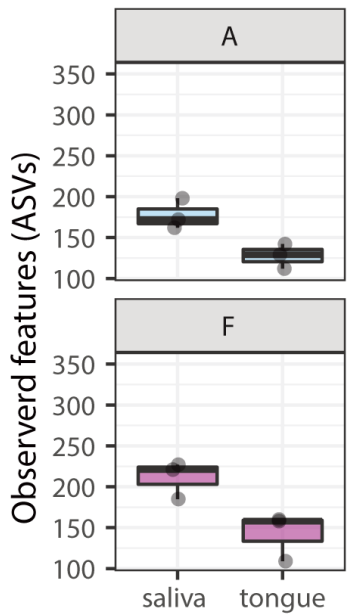

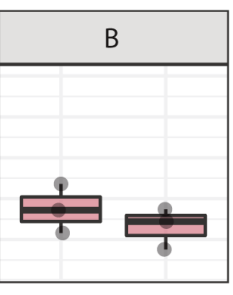

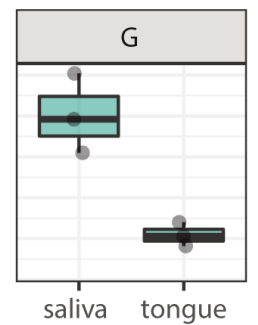

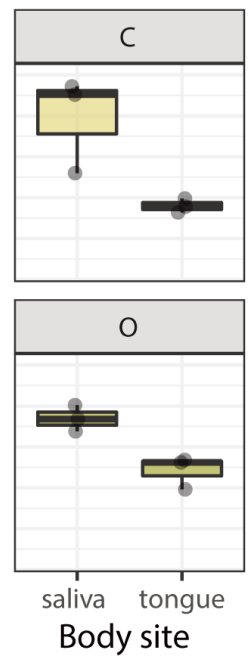

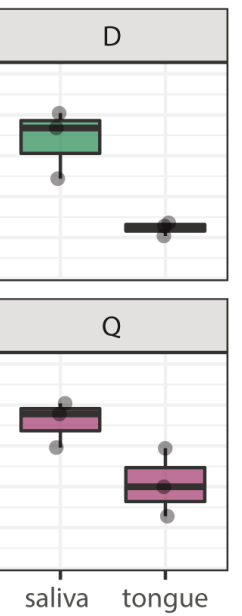

saliva tongue
Participant $\because \mathrm{A} \bullet \mathrm{C} \bullet \mathrm{E} \bullet \mathrm{G} \bullet \mathrm{Q}$

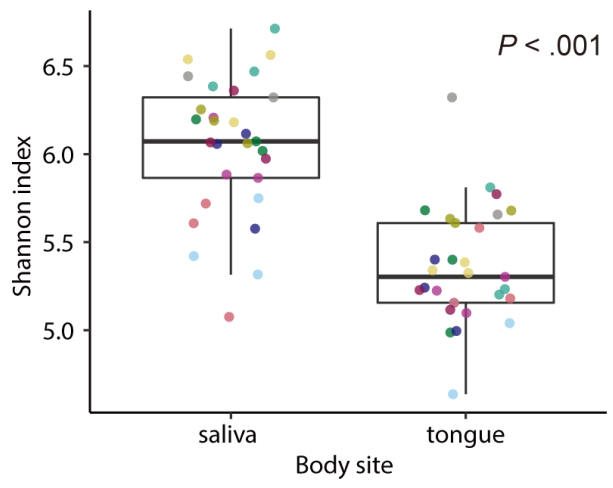

(b)

(c)

Figure 2. Alpha diversity of the salivary and tongue microbiomes. Data from the full amplicon sequence variants (ASV) table were used to calculate the alpha-diversity indexes. $(\mathbf{a}, \mathbf{b})$ Thirty samples each from the salivary or tongue microbiome were compared (three D1 samples for each of 10 participants). The number of observed ASVs (a) or Shannon index (b) was used as the alpha-diversity measure. Each point indicates a sample. Colors of the points indicate different participants. (c) Box plots of the number of observed ASVs in the salivary and tongue microbiomes ( $\mathrm{n}=3$ each) for each participant.

Porphyromonas $(6.1 \%$ and $6.7 \%$, respectively), and Fusobacterium $(4.6 \%$ and $7.6 \%$, respectively) (Figure $4 \mathrm{a})$. The abundance of bacterial taxa at species level is shown in Figure 4b. Prevotella melaninogenica HMT-469 was most abundant in both microbiomes $(8.1 \%$ in the saliva and $11.8 \%$ in the tongue), followed by Streptococcus oralis subsp. dentisani HMT-398 (7.2\%) and Haemophilus parainfluenzae HMT-718 $(7.0 \%)$ in the saliva, and by Fusobacterium periodonticum HMT-201 (7.4\%) and H. parainfluenzae HMT-718 (7.4\%) in the tongue (Figure 4b).

Five OTUs were differentially abundant in the salivary and tongue microbiomes $(P<0.05$, Kruskal-Wallis test) (Figure $4 \mathrm{c}$ ). Among them, S. oralis subsp. dentisani HMT-398 (7.2\% in the saliva and $2.7 \%$ in the tongue) and Neisseria mucosa HMT-682 (1.8\% and $0.2 \%$, respectively) were more abundant in the saliva, whereas F. periodonticum HMT-201 (3.5\% and 7.5\%, respectively), P. melaninogenica HMT-469 (8.1\% and $11.8 \%$, respectively), and Prevotella histicola HMT-298 (1\% and 2.1\%, respectively) were more abundant in the tongue (Figure $4 \mathrm{~b}$ and $4 c)$.

Core OTUs in the Salivary and Tongue Microbiomes To identify the core members of the oral microbiome, we focused on OTUs that were present in $\geq 95 \%$ of the saliva or tongue D1 samples. Seven OTUs were present in $\geq 95 \%$ of both, the saliva and tongue samples. These were $F$. periodonticum 


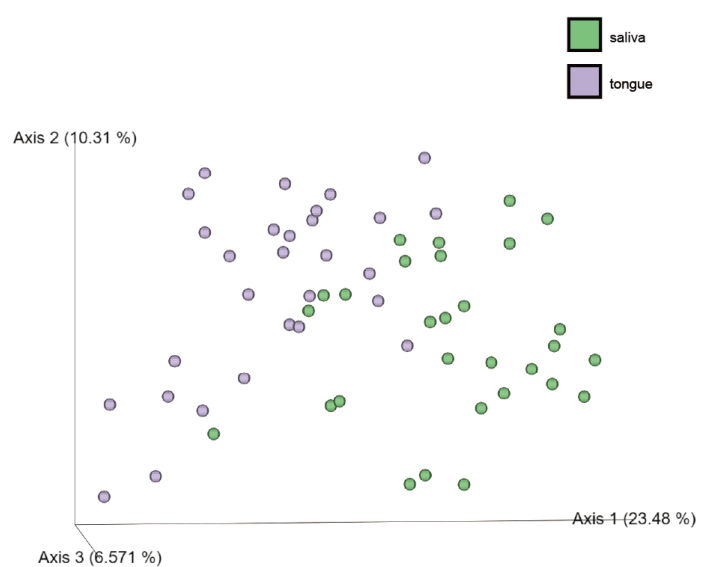

(a)

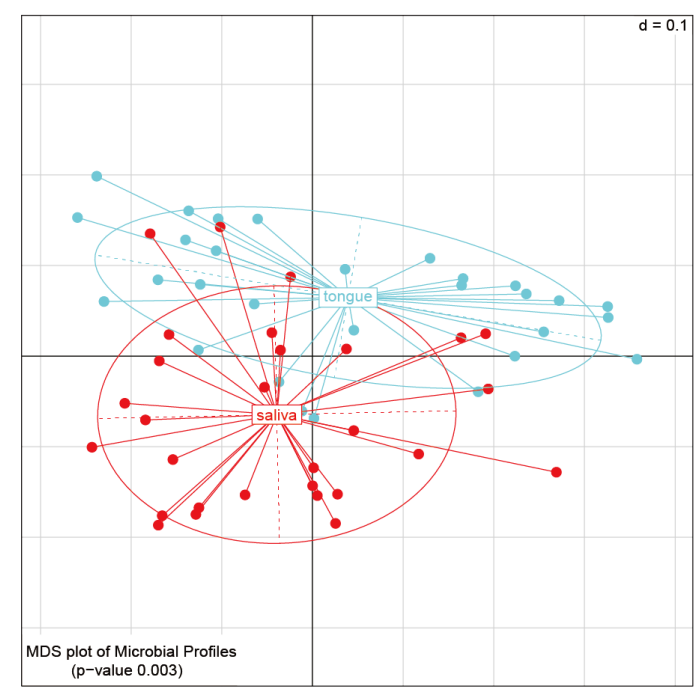

(c)

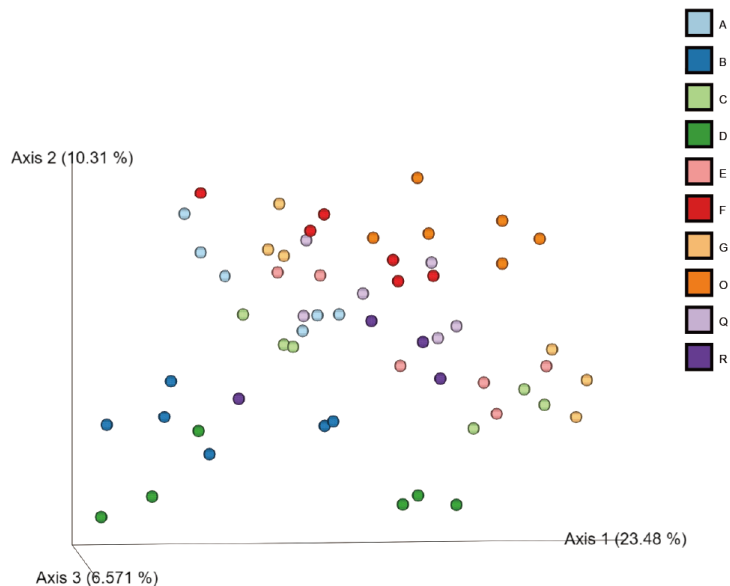

(b)

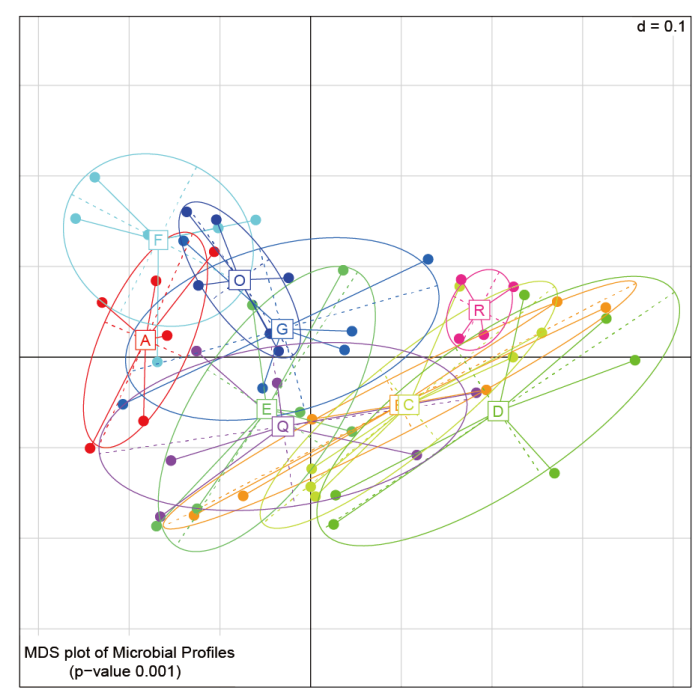

(d)

Figure 3. Beta diversity of the salivary and tongue microbiomes. (a, b) Three-dimensional principle co-ordinate analysis (PCOA) plots were generated with EMPeror using the full amplicon sequence variants (ASV) table. While only samples corresponding to D1 are displayed $(n=58)$, the PCoA analysis included all (116) samples. (c, d) multidimensional screening (MDS) plot of microbial profiles calculated based on generalized UniFrac distances using the clustered operational taxonomic unit (OTU) table. Each point indicates a sample. The points are colored according to the sampling site $(\mathbf{a}, \mathbf{c})$ or the participant from whom the sample was obtained $(\mathbf{b}, \mathbf{d})$.

HMT-201, Saccharibacteria (TM7) [G-1] sp. HMT-352, S. oralis subsp. dentisani HMT-398, P. melaninogenica HMT-469, Granulicatella adiacens HMT-534, Campylobacter concisus HMT-575, and H. parainfluenzae HMT-718 (Figure 5).

Further, we identified site-specific core OTUs, detected in $\geq 95 \%$ samples from one site but not from the other. The saliva-specific core OTUs were Veillonella parvula HMT-161, Porphyromonas pasteri HMT-279, Prevotella nanceiensis HMT-299, Gemella haemolysans HMT-626, and Alloprevotella sp. HMT-914. The tongue-specific core OTUs were Catonella morbi HMT-165, Oribacterium sinus HMT-457, Peptostreptococcaceae [XI][G-1] sulci HMT-467, Solobacterium moorei HMT-678, and Rothia mucilaginosa HMT-681 (Figure 5).

Effect of tablet taking on the salivary and tongue microbiomes

Using the above data as the base line, we finally assessed the effect of taking oral tablets (with or without protease) on the salivary and tongue microbiomes. Alpha diversity in D1 and D2 samples was not significantly different between any treatments (Figure 6a). In the control (no tablet) treatment, whereas 


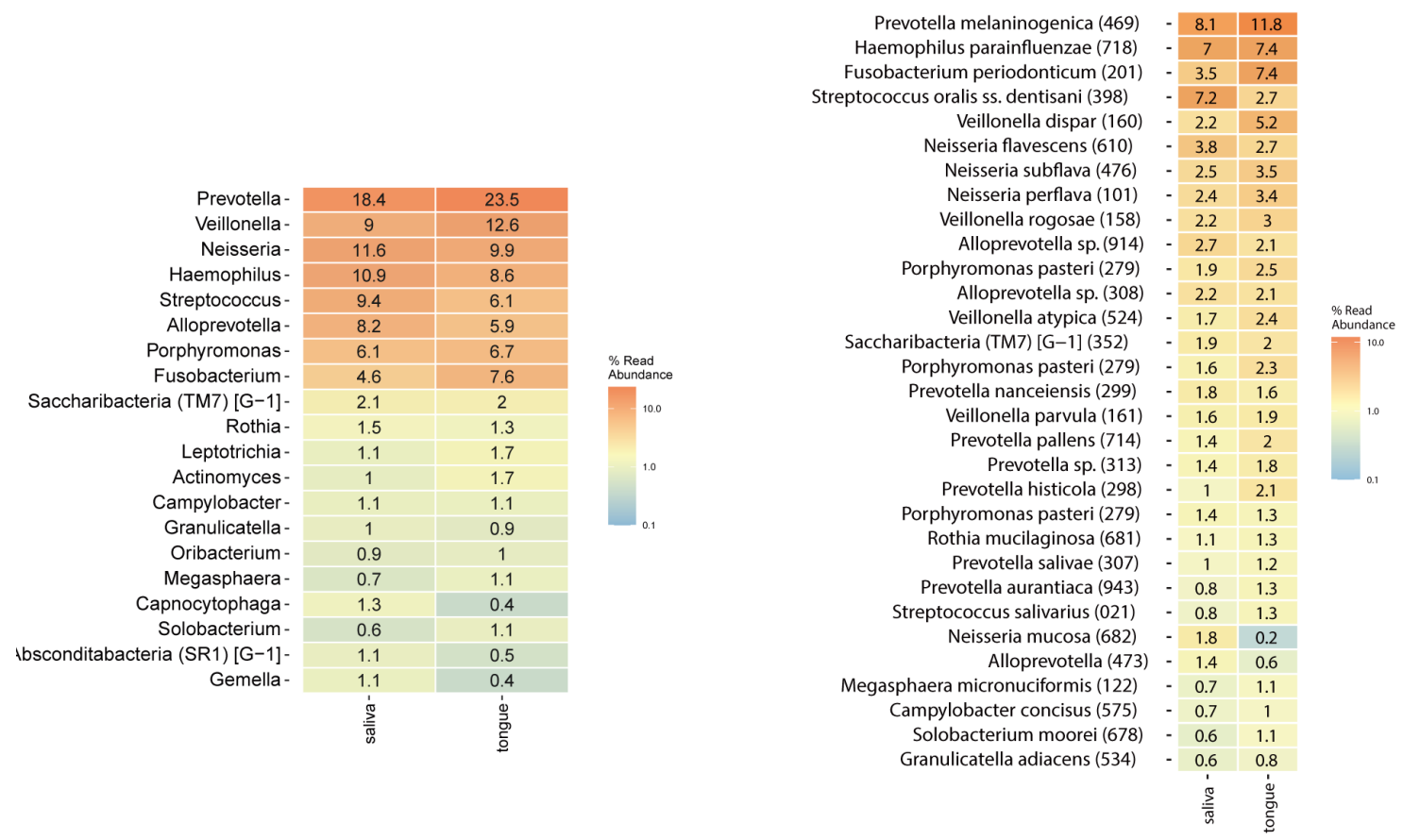

(a)

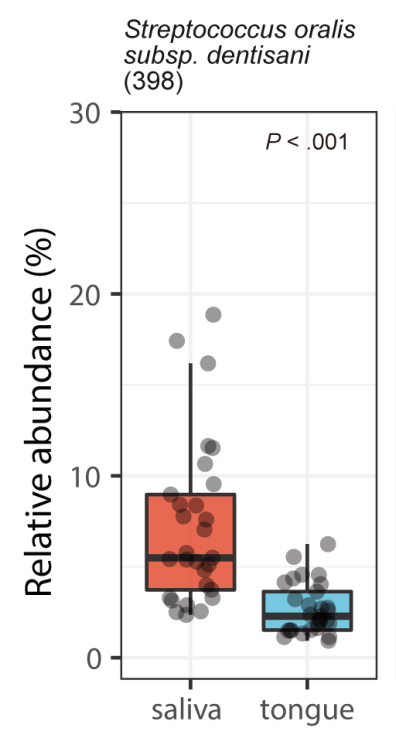

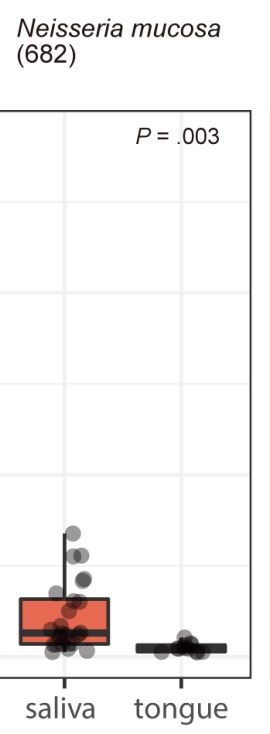

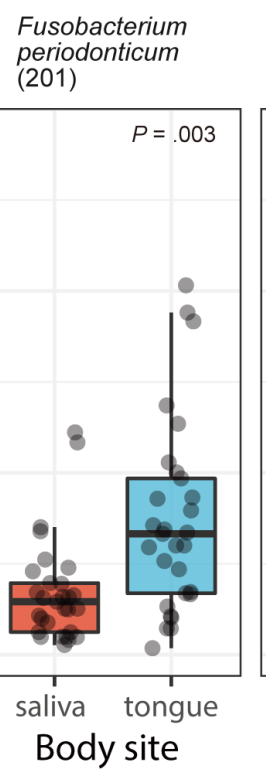

periodonticum $(201)$

(c) (b)

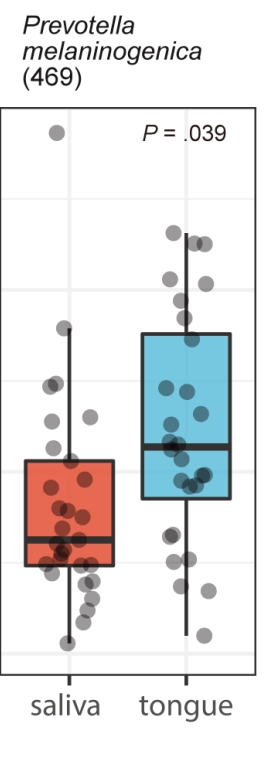

Prevotella histicola (298)

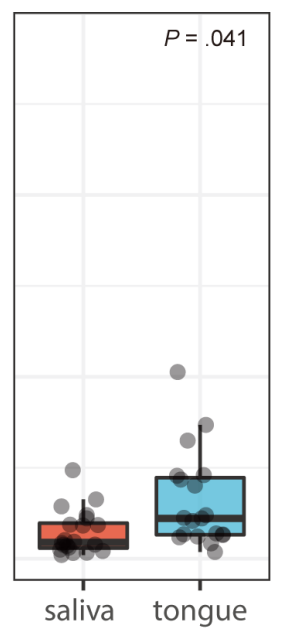

Figure 4. Abundances of bacterial taxa in the salivary and tongue microbiomes. The clustered operational taxonomic unit (OTU) table was used for calculations. (a, b) Heatmaps of the abundance of bacterial taxa at the genus (a) or species (b) levels. The \% read abundances are indicated, together with a color gradient. (c) Box plots summarizing the abundance of OTUs that were differentially abundant $(P<.05$, Kruskal-Wallis test with Benjamini-Hochberg adjustment) in the saliva and tongue samples at the species level are shown. Each dot indicates a sample. Taxon IDs in eHOMD are indicated in the parentheses.

the observed number of ASVs seemed to slightly increase in the saliva and to slightly decrease in the tongue, they were not statistically significant (Figure 6a). This indicates that some fluctuation of the oral microbiome may occur naturally. Further,
MDS analysis indicated that the beta diversity between D1 and D2 samples was not significantly different in any treatment, for either the salivary or tongue microbiome $(P>0.7$, Kruskal-Wallis test) (Figure 6B). 


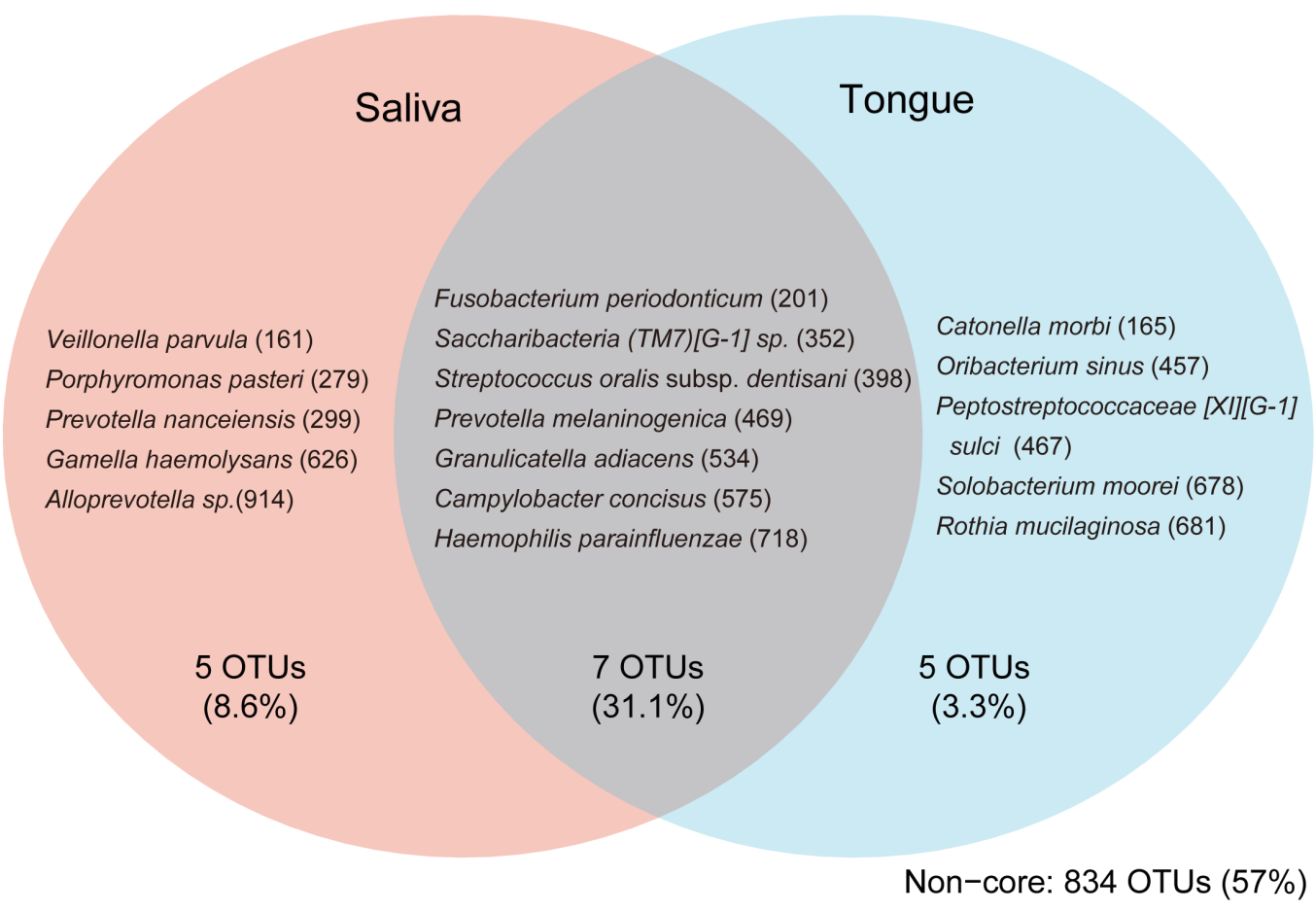

Figure 5. Venn diagram of the core oral operational taxonomic units (OTUs). OTUs present in $\geq 95 \%$ of samples (D1 samples, $n=58$ ) in the indicated subset of oral sites are shown together with the average total abundance of the OTUs in the group. For example, Veillonella parvula was found in $\geq 95 \%$ of saliva samples and $<95 \%$ of tongue samples. Taxon IDs in eHOMD are indicated in the parentheses.

We next examined whether any bacterial species were specifically impacted by oral tablet usage. Both ANCOM using the full ASV table or Kruskal-Wallis test using the clustered OTU table revealed that no OTU was differentially abundant before (D1) or after (D2) tablet use, in any of the treatments (no tablet, protease tablet, and plain tablet). The OTU abundance in each treatment group is summarized in Figure $7 a-c$. Although according to a recent study oral tablet use decreases the abundance of Fusobacterium nucleatum on the tongue of healthy young adults ${ }^{16}$, we did not detect any significant decrease of OTUs that correspond to $F$. nucleatum. Further, in the current study, whereas $7.6 \%$ of all OTUs from the tongue microbiome were assigned to the genus Fusobacterium (Figure $4 \mathrm{a}$ ), the majority of them were classified as $F$. periodonticum ( $7.5 \%$ of total) and only $<0.1 \%$ of all OTUs was assigned to F. nucleatum at species level.

\section{Discussion}

The oral microbiota has been associated with specific diseases in susceptible populations. In the current study, we examined the effect of oral care tablet use, with or without actinidin, on the salivary and tongue microbiomes. We showed that whereas there are some differences between the tongue and salivary microbiomes, the microbiomes were not affected by the oral tablet use, regardless of the tablet type. This does not preclude the possibility that a persistent oral tablet use would alter the oral microbiome. Controlled alteration of the oral microbiome has potential for disease prevention.
We here identify the core OTUs that are common between saliva and tongue. Among these OTUs, $S$. oralis and Campylobacter sp. have been determined to be the core OTUs common in the saliva and tongue previously ${ }^{3}$. F. periodonticum and Granulicatella adiacens have been found in tongue microbiome of adult, $P$. melaninogenica and $H$. parainfluenzae have been found in infant and adult tongue in common ${ }^{38}$. TM7 species have been identified in oral microbiomes including tongue ${ }^{39,40}$ and supragingival plaque ${ }^{41}$. It should be noted that although the high prevalence of Saccharibacteria (TM7) [G-1] sp. HMT-352 ( $\geq 95 \%$ in both saliva and tongue) as shown in our present study has not been reported previously, it could also be a result of clustering similar sequences into a single OTU. Although there are some differences in the classification of the core or predominant oral OTUs between the current and other studies $^{1,3}$, the majority of the species were identified as the core oral OTUs across the studies. Since low-abundance rather than highly abundant OTUs may contribute more to the difference in oral bacterial communities ${ }^{42,43}$, detailed analysis of low-abundance OTUs would be important in future research. $O$. sinus and $S$. moorei were previously classified as core OTUs common to the salivary and tongue microbiomes ${ }^{3}$, but in our present study were identified as tongue-specific. This seems reasonable considering that $S$. moorei plays an important role in halitosis ${ }^{4-46}$. The effective separation of saliva- and tongue-specific OTUs suggest the usefulness of our study design in analyzing the salivary and tongue microbiomes simultaneously. 

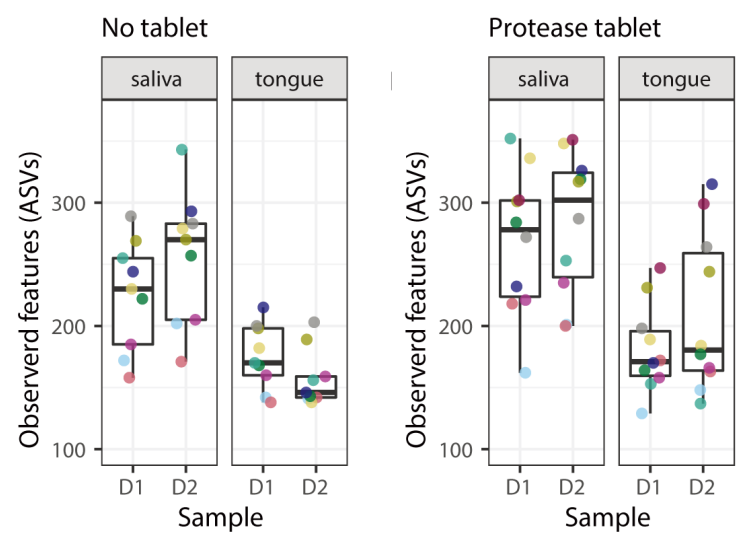

(a)
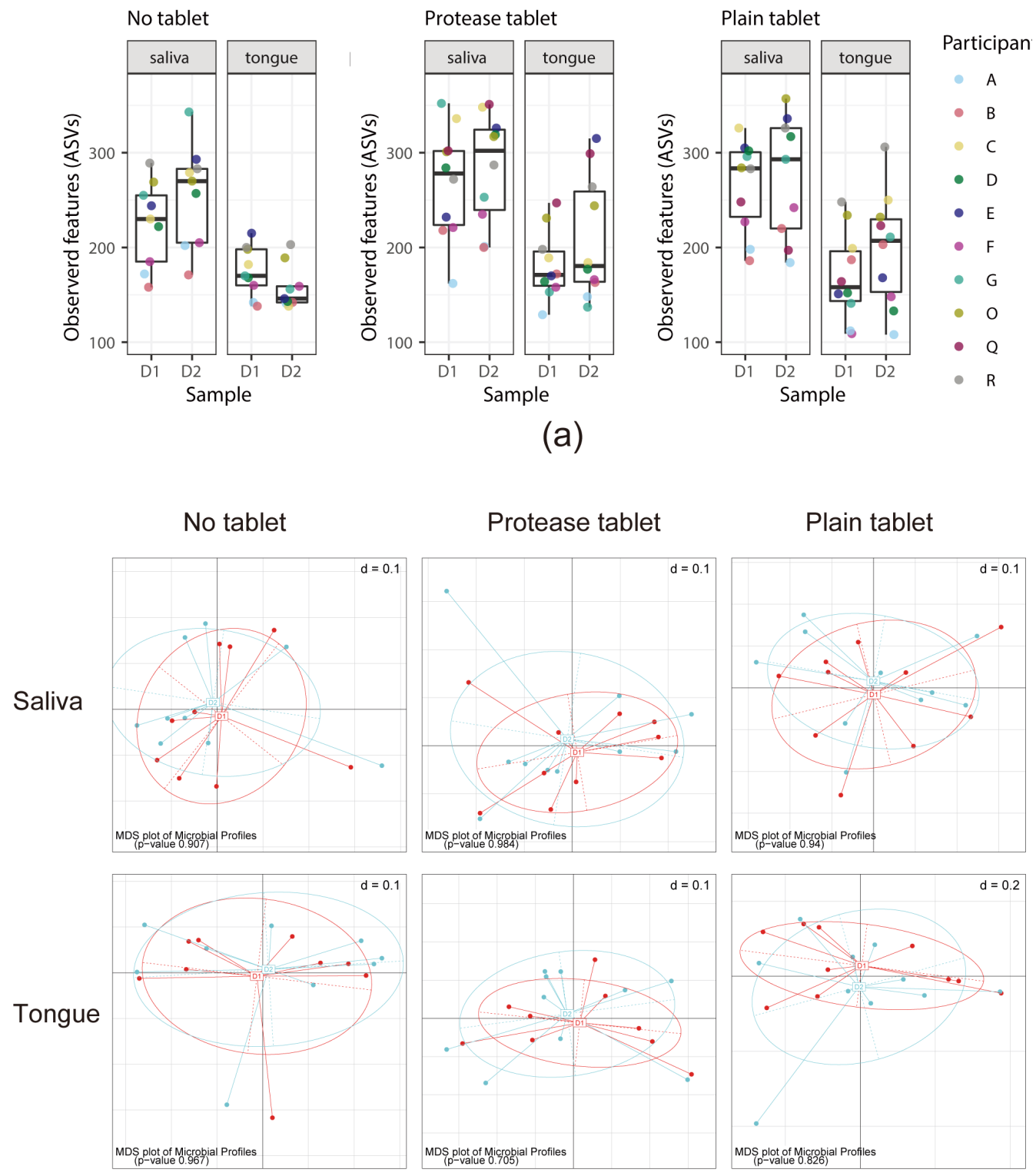

(b)

Figure 6. Effect of tablets on alpha and beta diversities of the salivary and tongue microbiomes. (a) Box plots of the number of observed amplicon sequence variants (ASV) in the saliva and tongue D1 and D2 samples in the no tablet (left), protease tablet (middle), and plain tablet (right) treatments. Each point indicates a sample. (b) multidimensional screening (MDS) analysis of beta diversity in D1 and D2 samples in the saliva (top panels) and tongue (bottom panels) in no tablet (left), protease tablet (middle), and plain tablet (right) treatments.

Our present study shows a variety among individuals in the number of observed ASVs in the salivary and tongue microbiomes. On the contrary, a significant interpersonal diversity in the supragingival plaque and salivary microbiomes, but not in the tongue plaque microbiomes was previously reported, using Faith's phylogenetic diversity as a measure ${ }^{3}$. Since the same V3-V4 region was targeted for amplicon sequencing in both studies, the discrepancy concerning the interpersonal differences in tongue microbiome might be associated with the differences in the alpha-diversity measure used, and/or in the participants' age $\left[25.3 \pm 3.1\right.$ years in Hall et al. study $^{3}$ and $39.8 \pm 10.9$ years (mean $\pm \mathrm{SD}$ ) in the current study (see Methods)].

The tongue microbe is associated with various diseases, including halitosis ${ }^{47,48}$ and aspiration pneumonia ${ }^{4}$; alterations in salivary microbiome has also linked to increasing numbers of oral and non-oral diseases ${ }^{49}$. With the advancement of microbiome studies, methods to predict host traits that predispose to 


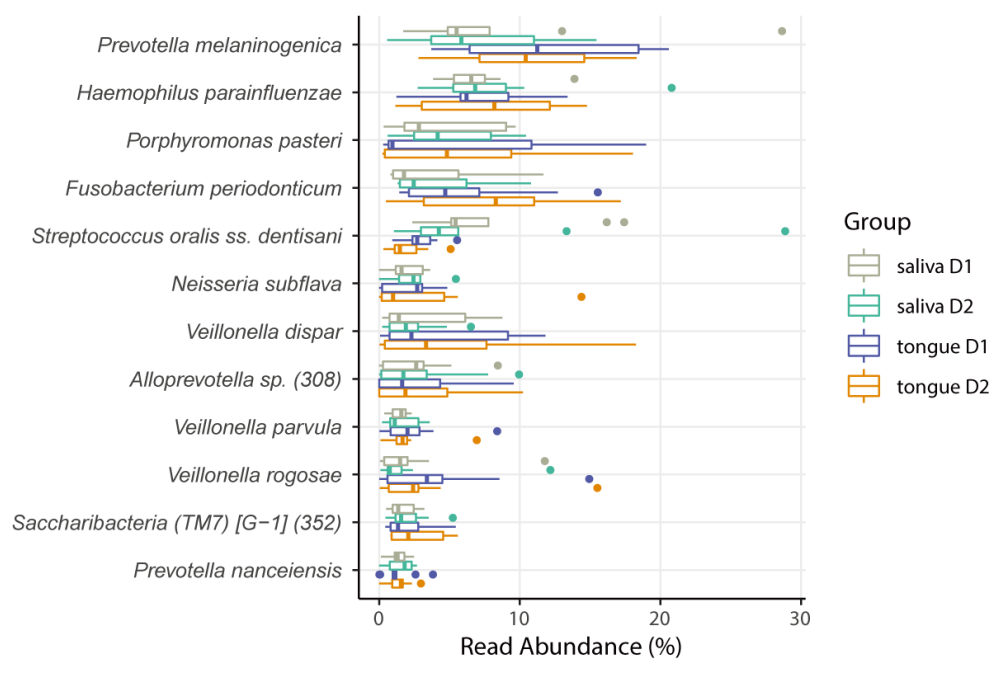

(a)

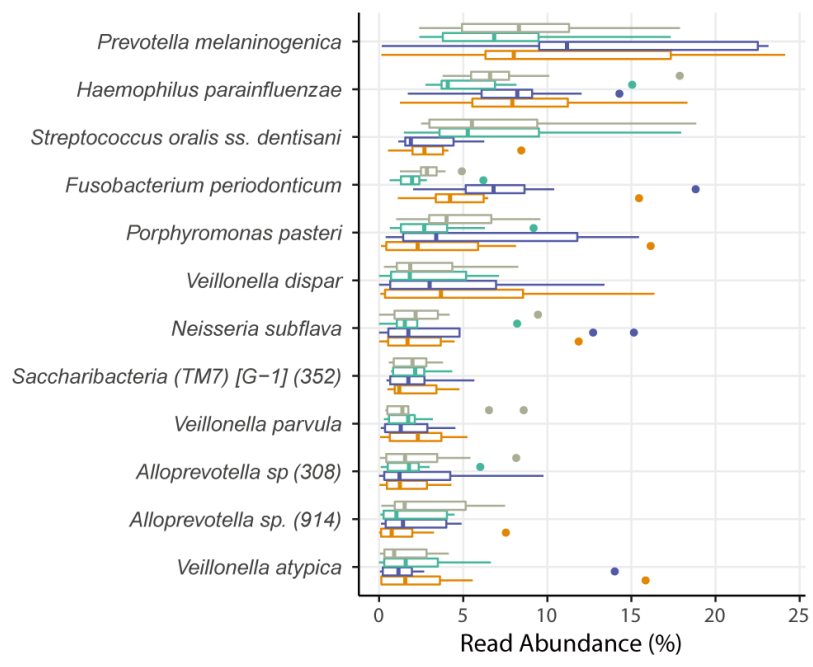

(b)

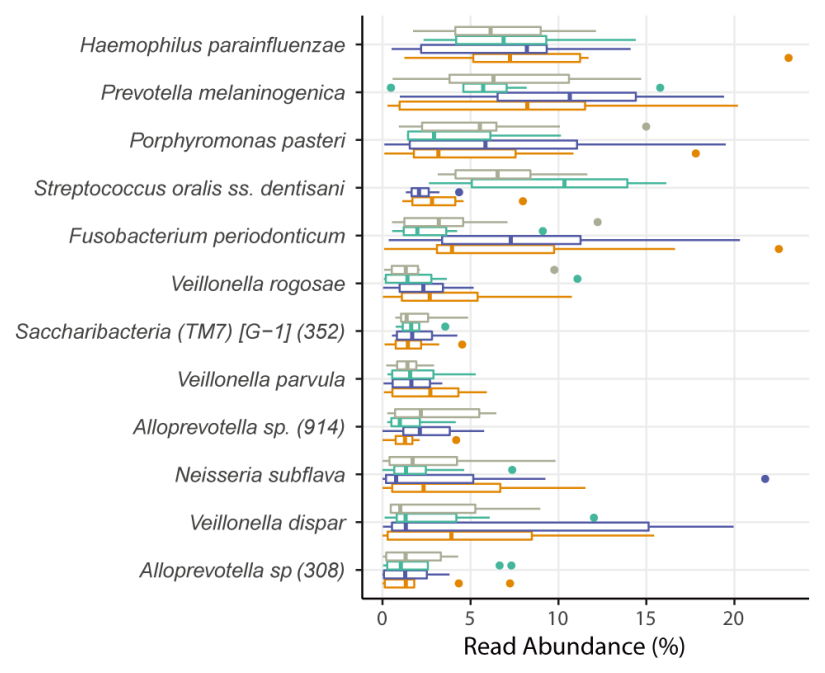

(c)

Figure 7. Box plots of OTU abundance. The abundances of top 12 operational taxonomic units (OTUs) based on the clustered OTU table for the no tablet (a), protease tablet (b), and plain tablet (c) treatments are shown. The data are colored depending on the group (saliva or tongue, and D1 or D2). No bacterial species showed differential abundance before (D1) or after (D2). Taxon IDs in eHOMD are indicated in the parentheses.

various diseases or conditions based on microbiome analysis have been developed ${ }^{50,51}$. Lu et al. have shown that tongue coating microbiome data can be used to distinguish individuals with pancreatic head carcinoma (PHC, one of pancreatic adenocarcinoma which occurs in the head of the pancreas) from healthy subjects ${ }^{39}$. Although the evaluation of an individual's disease status based on the tongue microbiota data is possible, the collection methods of the tongue coating samples may not be reliable when performed by a non-specialist, especially because of the anterior to posterior gradient of the bacterial communities in the tongue surface ${ }^{43}$. We here showed that the microbiomes of the saliva and tongue of an individual tend to be more similar to one another than to the salivary or tongue microbiomes from other individuals. Considering this fact together with the stability of oral microbiome over a prolonged period of time ${ }^{37}$, salivary collection could perhaps be used in the future as a standard method to predict diseases associated with the tongue-coating microbiota, as well as those linked to that of the saliva.

Oral care tablets have been shown previously to reduce tongue coating load and $\mathrm{VSCs}^{12,16}$. We here analyzed the effect of 
oral care tablets on the salivary and tongue microbiome. To avoid individual varieties in the amount of tongue coating or saliva flow affecting the analysis, we recruited only healthy adults to participate in this study. We did not detect any significant differences in the alpha diversity, beta diversity, or abundance of specific OTUs at species level after oral tablet use. There are several possible explanations for these observations. First, the participants of the current study were healthy adults with no apparent tongue coating accumulation. Although accumulated tongue coating can be reduced with oral tablets $^{12}$, the amount of tongue coating analyzed herein may have been insufficient for detecting the differences in the microbiota. Second, the tablet intervention period in the current study was only 1 day and the samples were collected 1 day after the tablet use. In contrast, twice daily tongue scraping for three days, together with sampling within 15 mins after intervention, have shown to reduce the gram-negative anaerobes on the tongue ${ }^{9}$. Although we chose to collect samples 1 day after the intervention, the 1-day period could have been long enough for the resilience of the oral microbiota to revert any shift in the oral microbiomes invoked by the tablet use. Considering these factors, analyzing oral care tablet intervention in individuals with a higher tongue coating index and/or over a longer period of time together with immediate sampling after intervention may provide more information on whether and how oral care tablets alter the oral microbiota, contributing to the maintenance of oral health.

Oral care tablets containing actinidin reduces tongue coating, and actinidin prevents biofilm formation by degrading cell-surface proteins in vitro ${ }^{12}$. We here attempted to elucidate the effect of the protease, supplied in oral tables, on the oral microbiome. Unfortunately, we were unable to assess the effect of the protease, because oral tablet treatments failed to alter the oral microbiome or specific bacterial taxa, regardless of the presence or absence of actinidin. As above, including participants with a higher tongue coating and a longer intervention period with immediate sampling may have allowed detection of the effect of actinidin in the tablets. Alternatively, an in vitro culturing system could be used to analyze the effect of actinidin on the oral microbiome, with the effects of the compound tested in a controlled manner. For example, nitric oxide ${ }^{17}$ or statins ${ }^{52}$ have been shown to alter the abundance of specific bacterial species. Using such system would allow the analysis of the effect of actinidin on oral microbiota separately from the effect of mechanical removing of the tongue coating.

Various lines of evidence suggest a link between oral microbiota and health or disease $\mathrm{s}^{1,2,53}$. The current and other ${ }^{3}$ studies have highlighted interpersonal differences in the oral microbiota. Several types of tongue microbiota have been shown to exist in individuals with different susceptibility to pneumonia ${ }^{4}$. Hence, personalized treatment based on an individual's oral microbiota is required, as has been already pointed out in the context of periodontal disease ${ }^{54}$. Analysis of how different types of oral microbiota are affected by certain interventions (e.g., oral care tablet or antibiotic treatment) would enable a more precise control over the oral microbiome in the future. In vitro culturing systems mentioned above are powerful tools for elucidating responses of bacterial communities taken from different individual to various interventions, and the contributing factors.

In conclusion, we have shown that while the salivary and tongue microbiomes differ significantly in terms of bacterial composition, they show inter- rather than intra-individual diversity. We have also identified bacterial species that are common to the salivary and tongue microbiome, as well as those that are specific to either of these. In addition, we showed that oral care tablets may not alter the bacterial composition of the saliva or the tongue, at least over short periods of time in healthy individuals. Considering the link between oral microbiota and health or disease, analyzing the differences in how individual oral microbiota responds to external factors will pave the way to more effective therapeutic and diagnostic approaches and, ultimately, contribute to the development of personalized dental medicine.

\section{Data availability}

\section{Underlying data}

Raw nucleotide sequences are available at DDBJ/EMBL-EBI/ NCBI database under the accession number DRA010849.

Figshare: Table_S1_sample-metadata.tsv for "Inter-site and interpersonal diversity of salivary and tongue microbiomes, and the effect of oral care tablets". https://doi.org/10.6084/ m9.figshare.13289618.v $1^{35}$

Table_S1_sample-metadata.tsv: Columns indicate sample ID, participant, sampling date (D1 or D2), treatment, Miseq run number, and sampling body site (saliva or tongue) of each sample. The "treatment" column indicates, no tablet (E2), protease tablet (E3), or plain tablet (E4) treatments.

Figshare: Table_S2_clustered-OTU-table.tsv for "Inter-site and interpersonal diversity of salivary and tongue microbiomes, and the effect of oral care tablets". https://doi.org/10.6084/ m9.figshare.13291535.v1 $1^{36}$

Table_S2_clustered-OTU-table. After open-reference clustering, OTU table was constructed from the BIOM file using QIIME 2. Number of reads for each OTU in each sample are indicated, together with the bacterial taxonomy assigned to each OTU. OTU IDs are identical to matching HOMD Refseq IDs, except for those which did not match the database.

Data are available under the terms of the Creative Commons Zero "No rights reserved" data waiver (CC0 1.0 Public domain dedication)

\section{Acknowledgments}

We would like to thank Hisako Sakamoto for preparation of DNA libraries, Makoto Taniguchi for Miseq sequencing, and Editage (www.editage.com) for English language editing. 
1. Yamashita Y, Takeshita T: The oral microbiome and human health. J Oral Sci. 2017: 59(2): 201-206.

PubMed Abstract | Publisher Full Text

2. Willis JR, Gabaldon T: The Human Oral Microbiome in Health and Disease: From Sequences to Ecosystems. Microorganisms. 2020; 8(2): 308. PubMed Abstract | Publisher Full Text | Free Full Tex

3. Hall MW, Singh N, Ng KF, et al.: Inter-personal diversity and temporal dynamics of dental, tongue, and salivary microbiota in the healthy oral cavity. NPJ Biofilms Microbiomes. 2017; 3: 2 . PubMed Abstract | Publisher Full Text | Free Full Text

4. Asakawa M, Takeshita T, Furuta M, et al.: Tongue Microbiota and Oral Health Status in Community-Dwelling Elderly Adults. mSphere. 2018; 3(4): e00332-00318.

PubMed Abstract | Publisher Full Text | Free Full Text

5. Van Tornout $M$, Dadamio J, Coucke $W$, et al.: Tongue coating: related factors. J Clin Periodontol. 2013; 40(2): 180-185. PubMed Abstract | Publisher Full Text

6. Yoneyama $\mathrm{T}$, Yoshida $\mathrm{M}$, Ohrui $\mathrm{T}$, et al:: Oral care reduces pneumonia in older patients in nursing homes. J Am Geriatr Soc. 2002; 50(3): 430-433. PubMed Abstract | Publisher Full Text

7. Müller F: Oral Hygiene Reduces the Mortality from Aspiration Pneumonia in Frail Elders. J Dent Res. 2015: 94(3 Suppl): 14S-16S. PubMed Abstract | Publisher Full Text | Free Full Text

8. Abe $\mathrm{S}$, Ishihara $\mathrm{K}$, Adachi $\mathrm{M}$, et al.: Tongue-coating as risk indicator for aspiration pneumonia in edentate elderly. Arch Gerontol Geriatr. 2008; 47(2): 267-275.

PubMed Abstract | Publisher Full Text

9. Bordas A, McNab R, Staples AM, et al.: Impact of different tongue cleaning methods on the bacterial load of the tongue dorsum. Arch Oral Biol. 2008; 53 Suppl 1: S13-S18.

PubMed Abstract | Publisher Full Text

10. Nohno K, Yamaga T, Kaneko N, et al.: Tablets containing a cysteine protease, actinidine, reduce oral malodor: a crossover study. J Breath Res. 2012; 6(1): 017107.

PubMed Abstract | Publisher Full Text

11. Laleman I, Koop R, Teughels W, et al.: Influence of tongue brushing and scraping on the oral microflora of periodontitis patients.J Periodontal Res. 2018; 53(1): 73-79.

PubMed Abstract | Publisher Full Text

12. Mugita N, Nambu T, Takahashi K, et al.: Proteases, actinidin, papain and trypsin reduce oral biofilm on the tongue in elderly subjects and in vitro. Arch Oral Biol. 2017; 82: 233-240.

PubMed Abstract | Publisher Full Text

13. Kageyama S, Takeshita T, Furuta M, et al.: Relationships of Variations in the Tongue Microbiota and Pneumonia Mortality in Nursing Home Residents. J Gerontol A Biol Sci Med Sci. 2018; 73(8): 1097-1102. PubMed Abstract | Publisher Full Text

14. Maruyama H, Masago A, Nambu T, et al.: Amplicon sequence variant-based oral microbiome analysis using QIIME 2. J Osaka Dent Univ. 2020; 54: 273-281. Publisher Full Text

15. Masago A, Maruyama $\mathrm{H}, \mathrm{Nambu} T$, et al.: Influence of tongue brushing on oral microbiome diversity. J Osaka Dent Univ. 2020; 54: 205-212.

16. Matsumura $Y$, Hinode $D$, Fukui $M$, et al.: Effectiveness of an oral care tablet containing kiwifruit powder in reducing oral bacteria in tongue coating: $A$ crossover trial. Clin Exp Dent Res. 2020; 6(2): 197-206.

PubMed Abstract | Publisher Full Text | Free Full Tex

17. Nambu T, Wang D, Mashimo C, et al.: Nitric Oxide Donor Modulates a Multispecies Oral Bacterial Community - An In Vitro Study. Microorganisms. 2019; 7(9): 353.

PubMed Abstract | Publisher Full Text | Free Full Text

18. Enomoto A, Nambu T, Kashiwagi $K$, et al.: Impact of short-term saliva storage at room temperature on the microbial composition. J Osaka Dent Univ. 2020; 54(1): 73-81.

Publisher Full Text

19. Bolyen E, Rideout JR, Dillon MR, et al.: Reproducible, interactive, scalable and extensible microbiome data science using QIIME 2. Nat Biotechnol. 2019; 37(8): 852-857.

PubMed Abstract | Publisher Full Text | Free Full Text

20. Callahan BJ, McMurdie PJ, Rosen MJ, et al:: DADA2: High-resolution sample inference from Illumina amplicon data. Nat Methods. 2016; 13(7): 581-583. PubMed Abstract | Publisher Full Text | Free Full Text

21. Bokulich NA, Kaehler BD, Rideout JR, et al.: Optimizing taxonomic classification of marker-gene amplicon sequences with QIIME 2's q2feature-classifier plugin. Microbiome. 2018; 6(1): 90. PubMed Abstract | Publisher Full Text | Free Full Text

22. Escapa IF, Chen T, Huang Y, et al.: New Insights into Human Nostril Microbiome from the Expanded Human Oral Microbiome Database (eHOMD): a Resource for the Microbiome of the Human Aerodigestive Tract. mSystems. 2018; 3(6): e00187-00118.

PubMed Abstract | Publisher Full Text | Free Full Text
23. Katoh $\mathrm{K}$, Misawa K, Kuma K, et al:: MAFFT: a novel method for rapid multiple sequence alignment based on fast Fourier transform. Nucleic Acids Res. 2002; 30(14): 3059-3066.

PubMed Abstract | Publisher Full Text | Free Full Text

24. Price MN, Dehal PS, Arkin AP: FastTree 2 - Approximately maximumlikelihood trees for large alignments. PLoS One. 2010; 5(3): e9490. PubMed Abstract | Publisher Full Text | Free Full Text

25. Rideout JR, Chase JH, Bolyen E, et al.: Keemei: cloud-based validation of tabular bioinformatics file formats in Google Sheets. GigaScience. 2016; 5: 27.

PubMed Abstract | Publisher Full Text | Free Full Text

26. Lozupone C, Knight R: UniFrac: a New Phylogenetic Method for Comparing Microbial Communities. Appl Environ Microbiol. 2005; 71(12): 8228-8235. PubMed Abstract | Publisher Full Text | Free Full Text

27. Vázquez-Baeza $Y$, Pirrung $M$, Gonzalez $A$, et al.: EMPeror: a tool for visualizing high-throughput microbial community data. GigaScience. 2013; 2(1): 16. PubMed Abstract | Publisher Full Text | Free Full Text

28. Anderson MJ: A new method for non-parametric multivariate analysis of variance. Austral Ecology. 2001; 26(1): 32-46. Publisher Full Text

29. Mandal S, Van Treuren W, White RA, et al:: Analysis of composition of microbiomes: a novel method for studying microbial composition. Microb Ecol Health Dis. 2015; 26: 27663

PubMed Abstract | Publisher Full Text | Free Full Text

30. Rideout JR, He Y, Navas-Molina JA, et al: Subsampled open-reference clustering creates consistent, comprehensive OTU definitions and scales to billions of sequences. PeerJ. 2014; 2: e545. PubMed Abstract | Publisher Full Text | Free Full Text

31. Wang LG, Lam TTY, Xu S, et al.: Treeio: An R Package for Phylogenetic Tree Input and Output with Richly Annotated and Associated Data. Mol Biol Evol. 2019; 37(2): 599-603.

PubMed Abstract | Publisher Full Text | Free Full Text

32. Chen J, Bittinger K, Charlson ES, et al.: Associating microbiome composition with environmental covariates using generalized UniFrac distances. Bioinformatics. 2012; 28(16): 2106-2113. PubMed Abstract | Publisher Full Text | Free Full Text

33. Lagkouvardos I, Fischer S, Kumar N, et al.: Rhea: a transparent and modular $\mathrm{R}$ pipeline for microbial profiling based on 16S rRNA gene amplicons. Peer]. 2017: 5: e2836.

PubMed Abstract | Publisher Full Text | Free Full Text

34. Andersen KS, Kirkegaard RH, Karst SM, et al.: ampvis2: an R package to analyse and visualise 16S rRNA amplicon data. bioRxiv. 2018. Publisher Full Text

35. Maruyama H, Masago A, Nambu T, et al:: Table_S1_sample-metadata. tsv for "Inter-site and interpersonal diversity of salivary and tongue microbiomes, and the effect of oral care tablets". Figshare. 2020. http://www.doi.org/10.6084/m9.figshare.13289618.v1

36. Maruyama H, Masago A, Nambu T, et al.: Table_S2_clustered-OTU-table. tsv for "Inter-site and interpersonal diversity of salivary and tongue microbiomes, and the effect of oral care tablets". Figshare. 2020. http://www.doi.org/10.6084/m9.figshare.13291535.v1

37. Utter DR, Mark Welch JL, Borisy GG: Individuality, Stability, and Variability of the Plaque Microbiome. Front Microbiol. 2016; 7: 564. PubMed Abstract | Publisher Full Text | Free Full Text

38. Kageyama S, Asakawa M, Takeshita T, et al.: Transition of Bacterial Diversity and Composition in Tongue Microbiota during the First Two Years of Life. mSphere. 2019; 4(3): e00187-19.

PubMed Abstract | Publisher Full Text | Free Full Text

39. Lu H, Ren $\mathrm{Z}$, Li A, et al.: Tongue coating microbiome data distinguish patients with pancreatic head cancer from healthy controls. J Oral Microbiol. 2019; 11(1): 1563409.

PubMed Abstract | Publisher Full Text | Free Full Text

40. Takeshita T, Kageyama S, Furuta M, et al:: Bacterial diversity in saliva and oral health-related conditions: the Hisayama Study. Sci Rep. 2016; 6: 22164. PubMed Abstract | Publisher Full Text | Free Full Text

41. Xiao C, Ran S, Huang Z, et al.: Bacterial Diversity and Community Structure of Supragingival Plaques in Adults with Dental Health or Caries Revealed by 16 S Pyrosequencing. Front Microbiol. 2016; 7: 1145. PubMed Abstract | Publisher Full Text | Free Full Text

42. Greenwood D, Afacan B, Emingil G, et al:: Salivary Microbiome Shifts in Response to Periodontal Treatment Outcome. Proteomics Clin Appl. 2020; 14(3): e2000011. PubMed Abstract | Publisher Full Text

43. Proctor DM, Fukuyama JA, Loomer PM, et al.: A spatial gradient of bacterial diversity in the human oral cavity shaped by salivary flow. Nat Commun. 2018; 9(1): 681. PubMed Abstract | Publisher Full Text | Free Full Text

44. Hampelska K, Jaworska MM, Babalska ZL, et al.: The Role of Oral Microbiota in Intra-Oral Halitosis. J Clin Med. 2020; 9(8): 2484. PubMed Abstract | Publisher Full Text | Free Full Text 
45. Tanabe S, Grenier D: Characterization of volatile sulfur compound production by Solobacterium moorei. Arch Oral Biol. 2012; 57(12): 1639-1643. PubMed Abstract | Publisher Full Text

46. Suzuki N, Yoneda M, Takeshita T, et al:: Induction and inhibition of oral malodor. Mol Oral Microbiol. 2019; 34(3): 85-96. PubMed Abstract | Publisher Full Text

47. Seerangaiyan $\mathrm{K}$, van Winkelhoff AJ, Harmsen HJM, et al.: The tongue microbiome in healthy subjects and patients with intra-oral halitosis. J Breath Res. 2017; 11(3): 036010 PubMed Abstract | Publisher Full Text

48. Ye $\mathrm{W}, \mathrm{Zhang} \mathrm{Y}, \mathrm{He} \mathrm{M}$, et al:: Relationship of tongue coating microbiome on volatile sulfur compounds in healthy and halitosis adults. J Breath Res. 2019; 14(1): 016005. PubMed Abstract | Publisher Full Text

49. Acharya $A$, Chan $Y$, Kheur $S$, et al:: Salivary microbiome in non-oral disease: A summary of evidence and commentary. Arch Oral Biol. 2017; 83: 169-173. PubMed Abstract | Publisher Full Text

50. Zhou YH, Gallins P: A Review and Tutorial of Machine Learning Methods for
Microbiome Host Trait Prediction. Front Genet. 2019; 10: 579. PubMed Abstract | Publisher Full Text | Free Full Text

51. McGeachie MJ, Sordillo JE, Gibson T, et al.: Longitudinal Prediction of the Infant Gut Microbiome with Dynamic Bayesian Networks. Sci Rep. 2016; 6 20359.

PubMed Abstract | Publisher Full Text | Free Full Text

52. Kaminska M, Aliko A, Hellvard A, et al.: Effects of statins on multispecies oral biofilm identify simvastatin as a drug candidate targeting Porphyromonas gingivalis. J Periodontol. 2019; 90(6): 637-646.

PubMed Abstract | Publisher Full Text | Free Full Text

53. Zarco M, Vess T, Ginsburg G: The oral microbiome in health and disease and the potential impact on personalized dental medicine. Oral Dis. 2012; 18(2): 109-120.

PubMed Abstract | Publisher Full Text

54. Schwarzberg K, Le R, Bharti B, et al.: The Personal Human Oral Microbiome Obscures the Effects of Treatment on Periodontal Disease. PLoS One. 2014 9(1): e86708.

PubMed Abstract | Publisher Full Text | Free Full Text 


\section{Open Peer Review}

\section{Current Peer Review Status:}

\section{Version 1}

Reviewer Report 01 March 2021

https://doi.org/10.5256/f1000research.30395.r79136

(C) 2021 Deo $\mathbf{P}$. This is an open access peer review report distributed under the terms of the Creative Commons Attribution License, which permits unrestricted use, distribution, and reproduction in any medium, provided the original work is properly cited.

\section{Priya Nimish Deo}

Department of Oral and Maxillofacial Pathology and Oral Microbiology, Bharati Vidyapeeth Deemed to be University Dental College and Hospital, Pune, India

It is a well designed study.

Effect of one day use of oral care tablets on oral microbiome was studied. Monitoring of the salivary and the tongue microbiomes is conducted simultaneously during an interrvention. Their results showed that the alpha diversity was higher in the saliva than on the tongue without intervention. The tablets did not affect the diversity not the abundance of specific species. Studies need to be carried out for longer duration to study the shifts in the composition of the microbiome upon intervention.

Is the work clearly and accurately presented and does it cite the current literature? Yes

Is the study design appropriate and is the work technically sound?

Yes

Are sufficient details of methods and analysis provided to allow replication by others? Yes

If applicable, is the statistical analysis and its interpretation appropriate? Yes

Are all the source data underlying the results available to ensure full reproducibility? Yes

Are the conclusions drawn adequately supported by the results? Yes

Competing Interests: No competing interests were disclosed. 
Reviewer Expertise: Oral microbiome and oral cancer

\section{I confirm that I have read this submission and believe that I have an appropriate level of expertise to confirm that it is of an acceptable scientific standard.}

Author Response 02 Apr 2021

Hugo Maruyama, Osaka Dental University, Japan

Thank you very much for your review and comment on our manuscript.

Studies need to be carried out for longer duration to study the shifts in the composition of the microt We agree that longer term study is needed to determine the impact of oral care tablets on the oral microbio

Competing Interests: No competing interests were disclosed.

Reviewer Report 01 March 2021

https://doi.org/10.5256/f1000research.30395.r79337

(C) 2021 Kunnath Menon R. This is an open access peer review report distributed under the terms of the Creative Commons Attribution License, which permits unrestricted use, distribution, and reproduction in any medium, provided the original work is properly cited.

\section{Rohit Kunnath Menon}

Division of Clinical Dentistry, School of Dentistry, International Medical University, Kuala Lumpur, Malaysia

\section{Comment 1}

Abstract: The results section should provide values for the diversity and the names of at least the most important core OTUs.

\section{Comment 2}

The authors may include a significant limitation of the study in the discussion section. Which is the sample size of ten individuals. The conclusions regarding the inter-participant differences rather than intra-individual variation is also previously well-established by studies with larger sample size. Hence it is ambitious to claim that this result is well-established with the small sample size.

\section{Comment 3}

Another significant limitation is the the lack of clarity in how the individuals were deemed healthy orally as well as systemically. . The absence of clinical data on the caries and periodontal health status of each participant needs to be explained. Previous research clearly shows the impact of oral diseases in determining the microbiome of the oral cavity especially saliva.

\section{Comment 4}


It is not surprising that one day treatment with the tablet did not significantly impact the temporal variation. Previous research has shown that even antibiotic treatment does not significantly impact salivary microbiome. The impact of the external agents on the microbiome should be discussed with inclusion of more of such previous investigations. The exclusion criteria of one month for antibiotic treatment should also be discussed with respect to previous available literature:

- R K Menon, A Gomez, B W Brandt, Y Y Leung, D Gopinath, R M Watt, W Crielaard, K E Nelson, M G Botelho. Long-term impact of oral surgery with or without amoxicillin on the oral microbiome-A prospective cohort study. Sci Rep. 2019 Dec 10;9(1):18761 ${ }^{1}$

Zaura, E. et al. Same Exposure but Two Radically Different Responses to Antibiotics: Resilience of the Salivary Microbiome versus Long-Term Microbial Shifts in Feces. mBio 6, e01693-01615 (2015) ${ }^{2}$

\section{Comment 5}

Please check the English grammar. For example: "We here analyzed the effect of oral care tablets on the salivary and tongue microbiome."

\section{References}

1. Menon R, Gomez A, Brandt B, Leung Y, et al.: Long-term impact of oral surgery with or without amoxicillin on the oral microbiome-A prospective cohort study. Scientific Reports. 2019; 9 (1).

Publisher Full Text

2. Zaura E, Brandt BW, Teixeira de Mattos MJ, Buijs MJ, et al.: Same Exposure but Two Radically Different Responses to Antibiotics: Resilience of the Salivary Microbiome versus Long-Term Microbial Shifts in Feces.mBio. 2015; 6 (6): e01693-15 PubMed Abstract | Publisher Full Text

Is the work clearly and accurately presented and does it cite the current literature? Yes

Is the study design appropriate and is the work technically sound? Yes

Are sufficient details of methods and analysis provided to allow replication by others? Yes

If applicable, is the statistical analysis and its interpretation appropriate? Yes

Are all the source data underlying the results available to ensure full reproducibility? Yes

Are the conclusions drawn adequately supported by the results? Yes

Competing Interests: No competing interests were disclosed.

Reviewer Expertise: oral microbiome 
I confirm that I have read this submission and believe that I have an appropriate level of expertise to confirm that it is of an acceptable scientific standard.

Author Response 02 Apr 2021

Hugo Maruyama, Osaka Dental University, Japan

Thank you very much for your review and comments on our manuscript. Below is a pointby-point response to your comments.

\section{Comment 1}

Abstract: The results section should provide values for the diversity and the names of at least the most important core OTUs.

We have included the values of alpha diversity and the names of the core OTUs in the abstract.

\section{Comment 2}

The authors may include a significant limitation of the study in the discussion section. Which is the sample size of ten individuals. The conclusions regarding the interparticipant differences rather than intra-individual variation is also previously wellestablished by studies with larger sample size. Hence it is ambitious to claim that this result is well-established with the small sample size.

The following statement has been added to the last paragraph of the Discussion: ", although it should be noted that the study has a limitation in the sample size of ten individuals."

\section{Comment 3}

Another significant limitation is the lack of clarity in how the individuals were deemed healthy orally as well as systemically. The absence of clinical data on the caries and periodontal health status of each participant needs to be explained. Previous research clearly shows the impact of oral diseases in determining the microbiome of the oral cavity especially saliva.

The following description has been added to the "Participants" section of the Methods: "According to the medical questionnaire, (1) none of the participants were undergoing or planning treatment for dental caries or periodontal disease, (2) there were no participants who were suffering from diabetes, chronic kidney disease, lung diseases, malignant tumors, etc., or who were visiting hospitals or taking medication, and (3) none of the participants experienced frequent thirst."

\section{Comment 4}

It is not surprising that one day treatment with the tablet did not significantly impact the temporal variation. Previous research has shown that even antibiotic treatment does not significantly impact salivary microbiome. The impact of the external agents on the microbiome should be discussed with inclusion of more of such previous investigations. The exclusion criteria of one month for antibiotic treatment should also be discussed with respect to previous available literature:

R K Menon, A Gomez, B W Brandt, Y Y Leung, D Gopinath, R M Watt, W Crielaard, K E Nelson, M G Botelho. Long-term impact of oral surgery with or without amoxicillin on 
the oral microbiome-A prospective cohort study. Sci Rep. 2019 Dec 10;9(1):187611

Zaura, E. et al. Same Exposure but Two Radically Different Responses to Antibiotics: Resilience of the Salivary Microbiome versus Long-Term Microbial Shifts in Feces. mBio 6, e01693-01615 (2015)2

The following description has been added to the "Participants" section of the Methods: "The exclusion criteria of one month for antibiotic treatment was set based on previous reports on the robustness and resilience of salivary microbiome [Zaura, E. et al. 2019;Menon RK et al. 2015]. For example, change in microbiome caused by exposure to clindamycin lasted up to 1 month in saliva [Zaura, E. et al.]."

In addition, the following statement has been added to the sixth paragraph of the Discussion: "The impact of external agents on microbiome depends on the location of microbiome. For example, salivary microbiome is highly resilient against external agents including antimicrobials, compared to feces microbiome that is more easily affected [Zaura et al. mBio 6, e01693-01615 (2015)2]."

\section{Comment 5}

Please check the English grammar. For example: "We here analyzed the effect of oral care tablets on the salivary and tongue microbiome."

The text has been changed to "Here, we analyzed the effect of oral care tablets on the salivary and tongue microbiomes." The entire manuscript has been checked by a native speaker.

Competing Interests: No competing interests were disclosed.

Reviewer Report 22 December 2020

\section{https://doi.org/10.5256/f1000research.30395.r76292}

(C) 2020 Sato T. This is an open access peer review report distributed under the terms of the Creative Commons Attribution License, which permits unrestricted use, distribution, and reproduction in any medium, provided the original work is properly cited.

\section{Takuichi Sato}

Division of Clinical Chemistry, Niigata University Graduate School of Health Sciences, Niigata, Japan

\section{Suggestions:}

Results

Figure 4(b): The authors need to check the figure. There are THREE "Porphyromonas pasteri (279)" in the figure.

Discussion

Figure 5 should be cited in the second paragraph of the discussion. 
Typographical errors;

Results

P. 6: "Neisseria (11.6\% and $9.9 \%$ respectively)"

Insert a comma between "9.9\%" and "respectively".

P. 7: "F. periodonticum HMT-201 (3.5\% and 7.5\%, respectively."

"7.5\%" should read "7.4\%".

Figure 4(a): The authors need to check the figure. Absconditabacteria?

References:

The authors need to check the style of the references $\# 2,4,7,13,17,22,26,31,37,38,41,44,50$,

51 and 54. (Do not use capital letters in the title of each reference.)

Is the work clearly and accurately presented and does it cite the current literature?

Yes

Is the study design appropriate and is the work technically sound?

Yes

Are sufficient details of methods and analysis provided to allow replication by others? Yes

If applicable, is the statistical analysis and its interpretation appropriate?

I cannot comment. A qualified statistician is required.

Are all the source data underlying the results available to ensure full reproducibility?

Yes

Are the conclusions drawn adequately supported by the results?

Yes

Competing Interests: No competing interests were disclosed.

Reviewer Expertise: Oral Microbiology

I confirm that I have read this submission and believe that I have an appropriate level of expertise to confirm that it is of an acceptable scientific standard.

Author Response 02 Apr 2021

Hugo Maruyama, Osaka Dental University, Japan

Thank you very much for your review and comments on our manuscript.

Below is a point-by-point response to your comments. 


\section{Suggestions:}

Results

Figure 4(b): The authors need to check the figure. There are THREE "Porphyromonas pasteri (279)" in the figure.

Thank you for pointing this out. This was due to the multiple slightly different reference $16 \mathrm{~S}$ rRNAs sequences present in the HOMD database used for open-reference clustering of ASVs into OTUs (http://www.homd.org/index.php?name=HOMD\&view=dynamic\&oraltaxonid=279 ). Because the original figure was created based on counts per OTU, the table contained three "Porphyromonas pasteri (279)". In the revised figure, we aggregated the counts for OTUs with a common Taxon ID (in this case, HMT-279).

An explanation was added to the figure legend: "In (b), count for clustered OTUs with common Taxon ID were aggregated."

\section{Discussion}

Figure 5 should be cited in the second paragraph of the discussion.

Figure 5 is now cited as recommended.

\section{Typographical errors;}

\section{Results}

P. 6: "Neisseria ( $11.6 \%$ and $9.9 \%$ respectively)"

Insert a comma between "9.9\%" and "respectively".

P. 7: "F. periodonticum HMT-201 (3.5\% and 7.5\%, respectively."

"7.5\%" should read "7.4\%".

All typographical errors that were pointed out have been corrected.

Figure 4(a): The authors need to check the figure. Absconditabacteria?

The problem of the left edge of the figure being cut off has been fixed.

\section{References:}

The authors need to check the style of the references $\# 2,4,7,13,17,22,26,31,37,38$, $41,44,50,51$ and 54. (Do not use capital letters in the title of each reference.)

The style of the references has been unified as pointed out.

Competing Interests: No competing interests were disclosed. 
The benefits of publishing with F1000Research:

- Your article is published within days, with no editorial bias

- You can publish traditional articles, null/negative results, case reports, data notes and more

- The peer review process is transparent and collaborative

- Your article is indexed in PubMed after passing peer review

- Dedicated customer support at every stage

For pre-submission enquiries, contact research@f1000.com 